\title{
Through-Wall Image Enhancement Using Fuzzy and QR Decomposition
}

\author{
Muhammad Mohsin Riaz $^{1}$ and Abdul Ghafoor ${ }^{2}$ \\ ${ }^{1}$ Centre for Advanced Studies in Telecommunication (CAST), Comsats, Islamabad, Pakistan \\ ${ }^{2}$ Department of Electrical Engineering, College of Signals, National University of Sciences and Technology (NUST), Islamabad, Pakistan
}

Correspondence should be addressed to Abdul Ghafoor; abdulghafoor-mcs@nust.edu.pk

Received 5 August 2013; Accepted 2 January 2014; Published 23 February 2014

Academic Editors: S. Bourennane and J. Marot

Copyright (C) 2014 M. M. Riaz and A. Ghafoor. This is an open access article distributed under the Creative Commons Attribution License, which permits unrestricted use, distribution, and reproduction in any medium, provided the original work is properly cited.

QR decomposition and fuzzy logic based scheme is proposed for through-wall image enhancement. QR decomposition is less complex compared to singular value decomposition. Fuzzy inference engine assigns weights to different overlapping subspaces. Quantitative measures and visual inspection are used to analyze existing and proposed techniques.

\section{Introduction}

Mapping of scenes behind obstacles (including building wall, rubbers, grass, etc.) using through-wall imaging (TWI) is an unfolded research domain. Different military and commercial applications (including antiterrorism, hostage rescue and surveillance, etc. [1]) can benefit from TWI. Beside other challenges, minimization of unwanted artifacts (clutters/noise) has enjoyed special importance over last few years [2-13]. These unwanted artifacts significantly decrease target detection and recognition capabilities.

Existing TWI image enhancement (clutter removal) techniques include background scene subtraction (only feasible if with and without target images are available) [2], spatial filtering (assuming wall homogeneity at low frequencies) [3], wall modeling and subtraction (requiring complex process for inhomogeneous walls) $[4,5]$ Doppler filtering (applicable for moving targets only) [6], image fusion (requiring multiple data of the same scene) [7], and statistical techniques [8-13].

In this paper, a TWI image enhancement (clutter reduction) technique using QR decomposition (QRD) and fuzzy logic is presented (preliminary results presented in [13]). Weights are assigned to different QRD subspaces using fuzzy inference engine. Simulation results evaluated using mean square error (MSE), peak signal to noise ratio (PSNR), improvement factor (IF), and visual inspection (based on miss detection (MD) and false detection (FD)) are used to verify the proposed scheme.

\section{Proposed Image Enhancement Using QRD}

Let the input image $M$ (having dimensions $G \times H$ ) be decomposed into different subspaces $\left(M_{\mathrm{cl}}, M_{\mathrm{tar}}\right.$, and $\left.M_{\mathrm{no}}\right)$ using singular value decomposition (SVD) as

$$
M=\underbrace{\sum_{g=1}^{l_{1}} s_{g} u_{g} v_{g}^{T}}_{M_{\mathrm{cl}}}+\underbrace{\sum_{g=l_{1}+1}^{l_{2}} s_{g} u_{g} v_{g}^{T}}_{M_{\mathrm{tar}}}+\underbrace{\sum_{g=l_{2}+1}^{G} s_{g} u_{g} v_{g}^{T}}_{M_{\mathrm{no}}},
$$

where $U$ and $V$ are singular vector matrices and $S$ contains singular values. As discussed in [13], conventional SVD for TWI image enhancement assumes that the target is limited to the second spectral component only; that is,

$$
M_{\mathrm{SVD}}=s_{2} u_{2} v_{2}^{T} \text {. }
$$

Besides the high computational complexity of SVD which is $4 G^{2} H+8 G H^{2}+9 H^{3}[14]$, the statement of target containment in the second spectral component is not always true. To cater the above issues, QRD and fuzzy logic based scheme is proposed. The image $M$ can be decomposed into an orthogonal unitary matrix $Q$ (having dimensions $G \times H$ and 
TABLE 1: Comparison analysis of QRD algorithms for TWI.

\begin{tabular}{|c|c|c|c|c|c|c|}
\hline \multirow{2}{*}{ Algorithms } & \multicolumn{4}{|c|}{ Accuracy } & \multirow{2}{*}{ Complexity } & \multirow{2}{*}{ Stability } \\
\hline & $M-Q R$ & $Q^{T} Q-I$ & $Q^{T} M-R$ & $M R^{-1}-Q$ & & \\
\hline CGS QR & $1.091 \times 10^{-16}$ & $1.310 \times 10^{-10}$ & $6.724 \times 10^{-14}$ & $9.913 \times 10^{-14}$ & $2 G H^{2}$ & Unstable \\
\hline MGS QR & $1.075 \times 10^{-16}$ & $4.922 \times 10^{-13}$ & $4.842 \times 10^{-14}$ & $1.083 \times 10^{-12}$ & $2 G H^{2}$ & Stable \\
\hline HT QR & $1.291 \times 10^{-15}$ & $3.795 \times 10^{-15}$ & $3.333 \times 10^{-16}$ & $1.263 \times 10^{-12}$ & $4 G^{2} H+2 G H^{2}+\frac{2}{3} H^{3}$ & Stable \\
\hline Givens QR & $7.532 \times 10^{-16}$ & $6.702 \times 10^{-15}$ & $2.711 \times 10^{-16}$ & $1.118 \times 10^{-12}$ & $8 G^{2} H+2 G H^{2}+\frac{2}{3} H^{3}$ & Stable \\
\hline
\end{tabular}

column vectors $q_{g}$ ) and an upper triangular matrix $R$ (having dimensions $G \times H$ and row vectors $\left.r_{g}\right)$, that is,

$$
M=Q R
$$

Table 1 shows the accuracy, stability, and complexity analysis of different QRD algorithms (classical and modified GramSchmidt (CGS, MGS), Givens decomposition, Householder transformation (HT), etc. [14]) for TWI.

Identical to SVD, the first subspace $M_{1}=q_{1} r_{1}$ represents wall clutters and rest subspaces contain targets and noise. Note that due to overlapping boundaries of targets and noise, it is difficult to extract target subspaces accurately. Foregoing in view, a weighting $\mathrm{QRD}$ based scheme is proposed to enhance targets. The enhanced image $M_{\mathrm{tar}}$ is

$$
M_{\mathrm{tar}}=\sum_{g=2}^{G} w_{g} q_{g} r_{g}
$$

where $w_{g}$ are weights applied to different subspaces. Fuzzy logic is used for the automatic weight assignment [15].

2.1. Input and Output MFs. Let $\xi_{g}=\left\|r_{g}\right\|$ and $\Delta \xi_{g}=\left\|r_{g}\right\|-$ $\left\|r_{g+1}\right\|$ be norms and norm differences, respectively. Note that high value of $\xi_{g}$ and $\Delta \xi_{g}$ the corresponding subspace $q_{g} r_{g}$ more likely contains target(s) and is therefore enhanced by applying heavy weights (and vise versa).

Three Gaussian membership functions (MFs) $\zeta_{X^{x}}\left(x_{1}\right)=$ $\exp \left(-\left(\left(c_{1}-\bar{c}_{1}^{(x)}\right) / \sigma_{1}^{(x)}\right)^{2}\right)$ and $(x \in$ \{High, Medium, Low $\left.\}\right)$ are defined for $\xi_{g}$. Similarly $\zeta_{Y^{y}}\left(x_{2}\right)=\exp \left(-\left(\left(c_{2}-\bar{c}_{2}^{(y)}\right) /\right.\right.$ $\left.\left.\sigma_{2}^{(y)}\right)^{2}\right)$ and $(y \in\{$ High, Medium, Low $\})$ are defined for $\Delta \xi_{g}$, where $\left\{c_{1}, c_{2}\right\} \in[0,1], \bar{c}_{1}^{(x)}, \bar{c}_{2}^{(y)}$ and $\sigma_{1}^{(x)}, \sigma_{2}^{(y)}$ are means and variances of fuzzy sets, respectively.

$K$-means algorithm [16] is used to adjust the fuzzy parameters. $\xi_{k}$ and $\Delta \xi_{h}$ are first clustered into three groups based on respective histograms. The means and variances, respectively, of each group are used as centers $\bar{c}_{1}^{(x)}, \bar{c}_{2}^{(y)}$ and spreads $\sigma_{1}^{(x)}, \sigma_{2}^{(y)}$ of MFs. Five equally spaced output MFs $\zeta_{Z^{z}}(d)=-\left(\left(d-\bar{d}^{(z)}\right) / \varrho^{(z)}\right)^{2}(z \in$ \{Very High, High, Medium, Low, Very Low $\})$, where mean $\bar{d}^{(z)}$ and variance $\varrho^{(z)}$ are used.
2.2. Product Inference Engine (PIE). Gaussian fuzzifier maps the input $\xi_{g}$ and $\Delta \xi_{g}$ as

$$
\zeta_{X Y}\left(c_{1}, c_{2}\right)=\exp \left\{-\left(\frac{c_{1}-\xi_{g}}{v_{1}}\right)^{2}\right\} \exp \left\{-\left(\frac{c_{2}-\Delta \xi_{h}}{v_{2}}\right)^{2}\right\},
$$

where $v_{1}$ and $v_{2}$ are parameters used for input noise suppression and are chosen as $v_{1}=2 \max _{x} \sigma_{1}^{(x)}$ and $v_{2}=2 \max _{y}^{3} \sigma_{2}^{(y)}$ [15].

Fuzzy IF-THEN rules for image enhancement are the following.

Rule 1: IF $\xi_{h}$ is $X^{\mathrm{High}}$ and $\Delta \xi_{h}$ is $Y^{\mathrm{High}}$, THEN $w_{h}^{\mathrm{PIE}}$ is $Z^{\text {Very High }}$.

Rule 2: IF $\xi_{h}$ is $X^{\mathrm{Med}}$ and $\Delta \xi_{h}$ is $Y^{\mathrm{High}}$, THEN $w_{h}^{\mathrm{PIE}}$ is $Z^{\text {High }}$.

Rule 3: IF $\xi_{h}$ is $X^{\mathrm{High}}$ and $\Delta \xi_{h}$ is $Y^{\mathrm{Med}}$, THEN $w_{h}^{\mathrm{PIE}}$ is $Z^{\text {High }}$.

Rule 4: IF $\xi_{h}$ is $X^{\mathrm{Med}}$ and $\Delta \xi_{h}$ is $Y^{\mathrm{Med}}$, THEN $w_{h}^{\mathrm{PIE}}$ is $Z^{\mathrm{Med}}$.

Rule 5: IF $\xi_{h}$ is $X^{\mathrm{High}}$ and $\Delta \xi_{h}$ is $Y^{\mathrm{Low}}$, THEN $w_{h}^{\mathrm{PIE}}$ is $Z^{\mathrm{Med}}$.

Rule 6: IF $\xi_{h}$ is $X^{\text {Low }}$ and $\Delta \xi_{h}$ is $Y^{\mathrm{Med}}$, THEN $w_{h}^{\mathrm{PIE}}$ is $Z^{\mathrm{Med}}$.

Rule 7: IF $\xi_{h}$ is $X^{\mathrm{Med}}$ and $\Delta \xi_{h}$ is $Y^{\mathrm{Low}}$, THEN $w_{h}^{\mathrm{PIE}}$ is $Z^{\text {Low }}$.

Rule 8: IF $\xi_{h}$ is $X^{\text {Low }}$ and $\Delta \xi_{h}$ is $Y^{\mathrm{High}}$, THEN $w_{h}^{\mathrm{PIE}}$ is $Z^{\text {Low }}$.

Rule 9: IF $\xi_{h}$ is $X^{\text {Low }}$ and $\Delta \xi_{h}$ is $Y^{\text {Low }}$, THEN $w_{h}^{\text {PIE }}$ is $Z^{\text {Very Low }}$.

The output of PIE using individual rule based inference, Mamdani implication, algebraic product for $t$-norm, and max operator for $s$-norm [15] is

$\zeta_{Z^{\prime}}\left(d_{g}\right)=\max _{\{x, y, z\}}\left[\sup _{\left\{c_{1}, c_{2}\right\}} \zeta_{X Y}\left(c_{1}, c_{2}\right) \zeta_{X^{x}}\left(c_{1}\right) \zeta_{Y^{y}}\left(c_{2}\right) \zeta_{Z^{z}}\left(d_{g}\right)\right]$. 
The weights $w_{g}^{\mathrm{PIE}}$ are then computed as

$$
w_{g}^{\mathrm{PIE}}=\frac{\sum_{z} \bar{d}^{(z)} \omega_{g}^{(z)}}{\sum_{z}=1 \omega_{h}^{(z)}}
$$

where $\omega_{g}^{(z)}$ is the height of $\zeta_{Z^{\prime}}\left(d_{g}\right)$ in output MFs [15].

2.3. Takagi-Sugeno (TS) Inference. In contrast to PIE, TS inference engine adjusts the output MFs using adaptive and/ or optimization techniques [17]. The TS rule-base (IF-THEN) for computing weights $w_{g}^{\mathrm{TS}}$ is

$$
\begin{gathered}
\text { IF } \xi_{g} \text { is } X^{j_{1}} \operatorname{AND} \Delta \xi_{g} \text { is } Y^{j_{2}} \\
\operatorname{THEN~} p^{\left(j_{1}+j_{2}-1\right)}=\left(\frac{1}{1+\exp \left\{-\xi_{g}\right\}+\exp \left\{-\Delta \xi_{g}\right\}}\right)^{j_{1}+j_{2}-1} .
\end{gathered}
$$

Note that the output reduces for large $j_{1}+j_{2}$ (which is desirable). The aggregated weights $w_{g}^{\mathrm{TS}}$ are

$$
w_{g}^{\mathrm{TS}}=\frac{\sum_{j_{1}=1}^{3} \sum_{j_{2}=1}^{3} p^{\left(j_{1}+j_{2}-1\right)} t\left\{\zeta_{X^{j_{1}}}\left(\xi_{k}\right), \zeta_{Y^{j_{2}}}\left(\Delta \xi_{g}\right)\right\}}{\sum_{j_{1}=1}^{3} \sum_{j_{2}=1}^{3} t\left\{\zeta_{X^{j_{1}}}\left(\xi_{g}\right), \zeta_{Y^{j_{2}}}\left(\Delta \xi_{g}\right)\right\}},
$$

where $t$ represents algebraic product (intersection operator).

\section{Simulation and Results}

Experimental setup for TWI is constructed using Agilent's vector network analyzer (VNA) which generates stepped frequency waveforms between $2 \mathrm{GHz}$ and $3 \mathrm{GHz}(1 \mathrm{GHz}$ band width $(\mathrm{BW}))$ having step size of $\Delta f=5 \mathrm{MHz}$ and step size $N_{f}=201$. Maximum range is $R_{\max }=30 \mathrm{~m}$ and range resolution is $\Delta R=0.15 \mathrm{~m}$.

Broadband horn antenna which is mounted on twodimensional scanning frame (having dimensions $2.4 \mathrm{~m} \times$ $3 \mathrm{~m}$ (width $\times$ height) and can slide along cross range and height) operates in monostatic mode with $12 \mathrm{~dB}$ gain. Thickness of the wall is $5 \mathrm{~cm}$ and relative permittivity and permeability are 2.3 and 1 , respectively. The frame is placed $0.03 \mathrm{~m}$ away from wall and scanning is controlled by a microcontroller based mechanism. The scattering parameters are recorded at each step and transferred to a local computer for image reconstruction and processing. Received data is converted into time domain and beamforming algorithm is used for image reconstruction. Existing and proposed image
TABLE 2: MSE, PSNR, IF, MD, and FD comparison.

\begin{tabular}{lcccccc}
\hline Scenario & Scheme & MSE & PSNR & IF & MD & FD \\
\hline \multirow{4}{*}{ Two targets } & SVD [11] & 0.2726 & 5.6442 & 8.1258 & 1 & 0 \\
& Fuzzy QRD (PIE) & 0.1970 & 7.0553 & 11.2587 & 0 & 0 \\
& Fuzzy QRD (TS) & 0.1726 & 7.6296 & 11.5870 & 0 & 0 \\
\hline \multirow{4}{*}{ Three targets } & Fuzzy QRD (PIE) & 0.1933 & 7.1377 & 10.8715 & 0 & 0 \\
& Fuzz [11] & 0.2814 & 5.5068 & 7.1265 & 1 & 1 \\
& Fuzd QRD (TS) & 0.1824 & 7.3898 & 11.0127 & 0 & 0 \\
\hline
\end{tabular}

enhancement algorithms are simulated in MATLAB and quantitative analysis is performed using MSE, PSNR, IF, FD, $\mathrm{MD}$, and visual inspection:

$$
\begin{gathered}
\text { MSE }=\frac{1}{G \times H} \sum_{g=1}^{G} \sum_{h=1}^{H}\left(M_{b s}(g, h)-M_{\mathrm{tar}}(g, h)\right)^{2}, \\
\operatorname{PSNR}(\mathrm{dB})=10 \log _{10} \frac{1}{\mathrm{MSE}}, \\
\operatorname{IF}(\mathrm{dB})=10 \log _{10}\left[\frac{P_{M_{\mathrm{tar}}, t} \times P_{M, c}}{P_{M, t} \times P_{M_{\mathrm{tar}}, c}}\right],
\end{gathered}
$$

where $M_{b s}$ is a reference image obtained by the difference of image, with and without target. $P_{M_{\mathrm{tar}}, t}$ and $P_{M_{\mathrm{tar}}, \mathrm{c}}$ are average pixel values of target and clutter in enhanced image, respectively. $P_{M, t}$ and $P_{M, c}$ are average pixel values of target and clutter in the original image, respectively.

MD is defined as "target was present in the original image, but was not detected in enhanced image." FD is defined as "target was not present in the original image, but was detected in enhanced image." For calculating FD and MD, a threshold is calculated using global thresholding algorithm [18].

Figure 1 shows the original B-scan containing two targets, the background subtracted reference and enhanced images, using existing SVD and proposed QRD based schemes. It can be observed that proposed schemes detect both targets whereas SVD based scheme is unable to locate both targets accurately.

Figure 2 shows another example containing three targets. The proposed scheme detects all targets and provides a better target to background ratio compared to SVD based scheme. It is further noted that the proposed TS inference based scheme provides better results compared to PIE.

Table 2 shows that proposed fuzzy QRD schemes are better (as compared to the SVD image enhancement scheme) in terms of MSE, PSNR, IF, MD, and FD.

\section{Conclusion}

QRD and fuzzy logic based image enhancement scheme is proposed for TWI. Compared with SVD, QRD provides less computational complexity. PIE and TS inference engines are used to assign weights to different QRD subspaces. Simulation results compared on visual and quantitative analysis show the significance of the proposed scheme. 


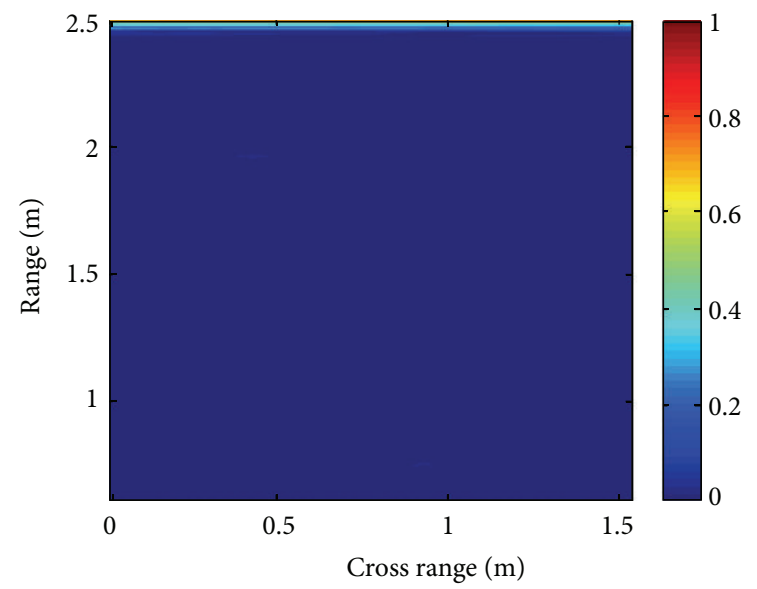

(a)

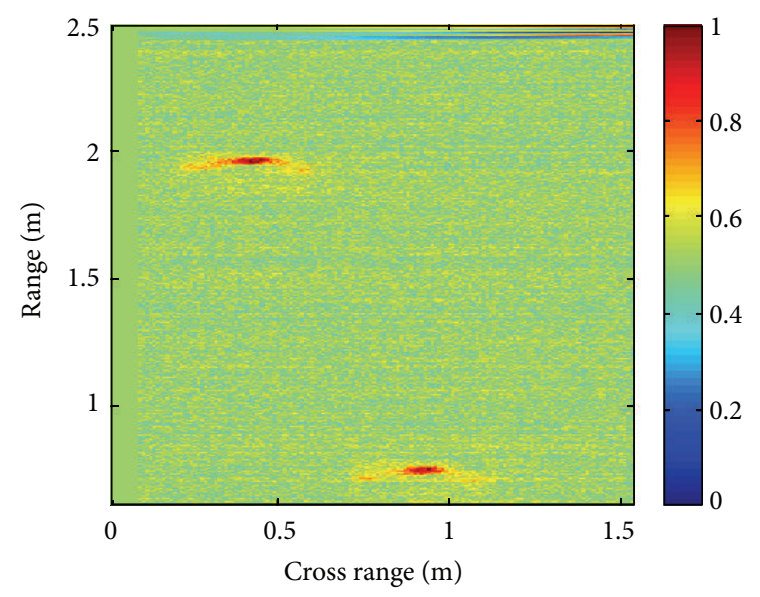

(c)

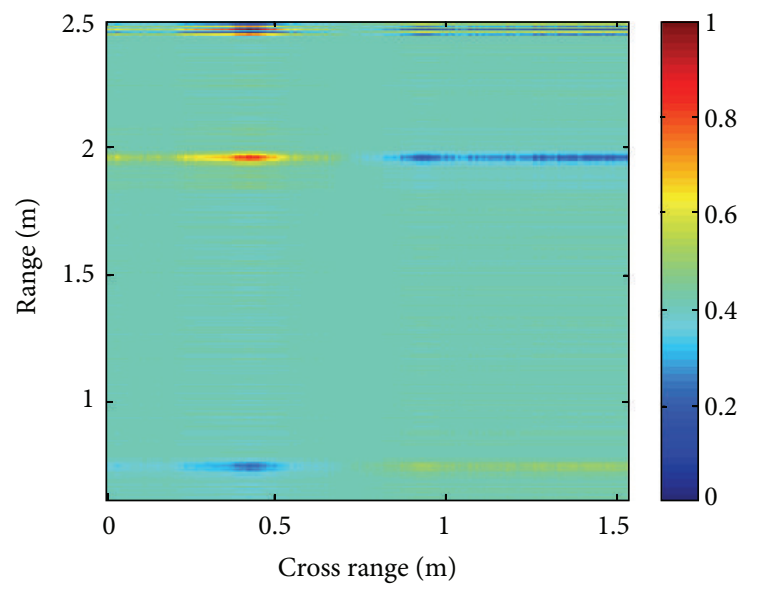

(b)

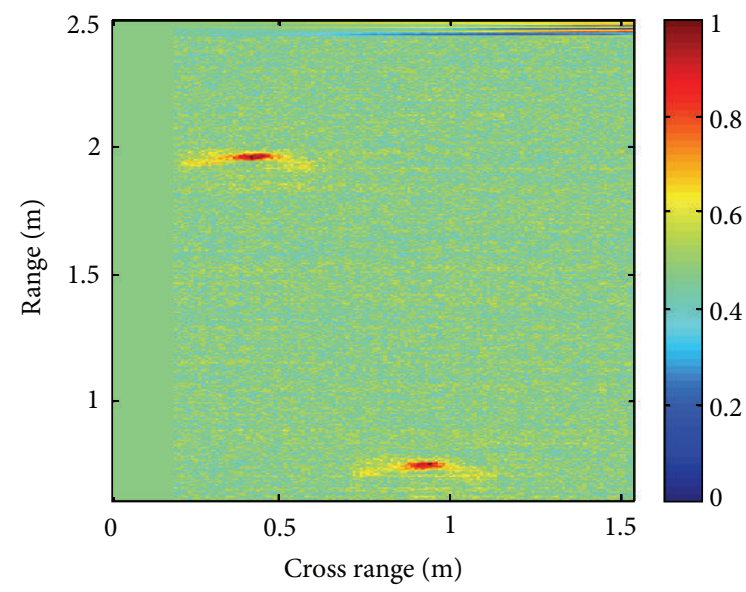

(d)

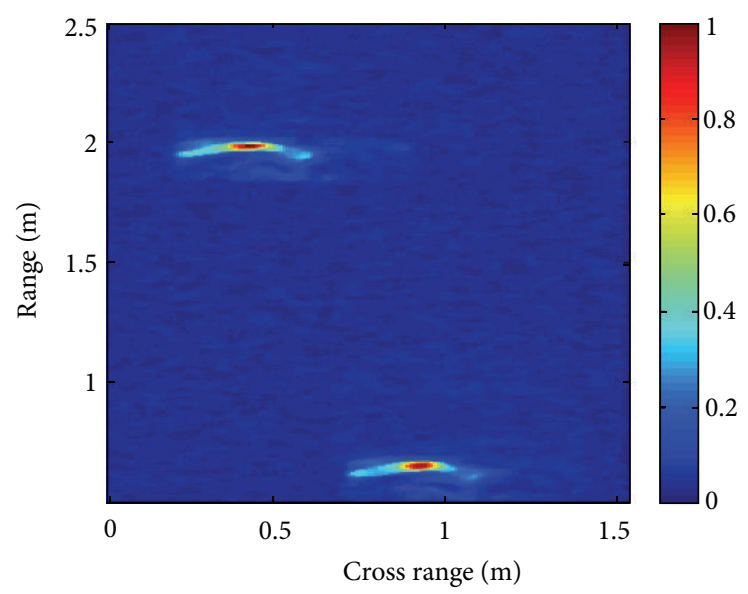

(e)

FIgure 1: Image with two targets. (a) Original image. (b) SVD [11]. (c) Proposed fuzzy QRD (PIE). (d) Proposed fuzzy QRD (TS). (e) Background subtracted reference image. 


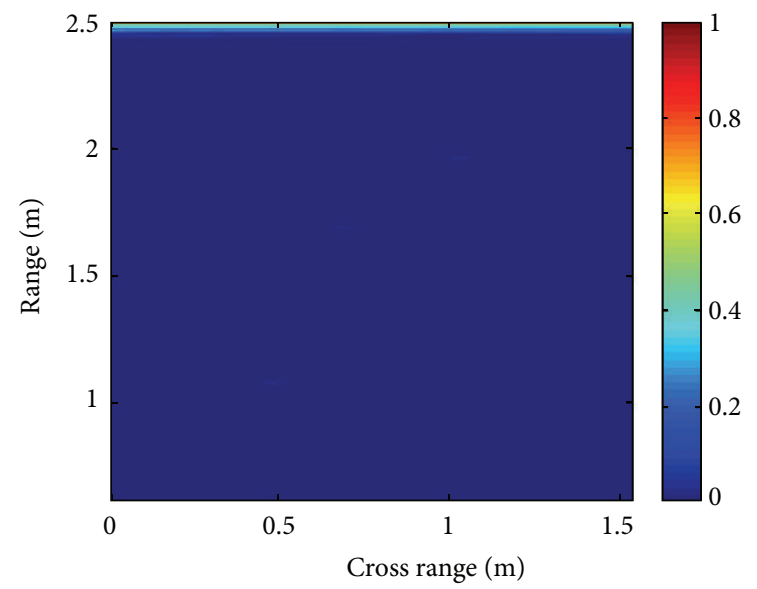

(a)

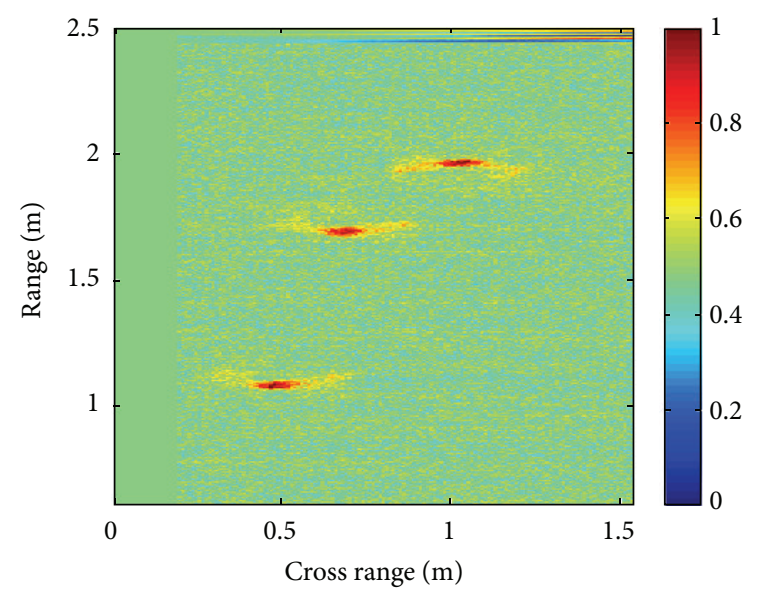

(c)

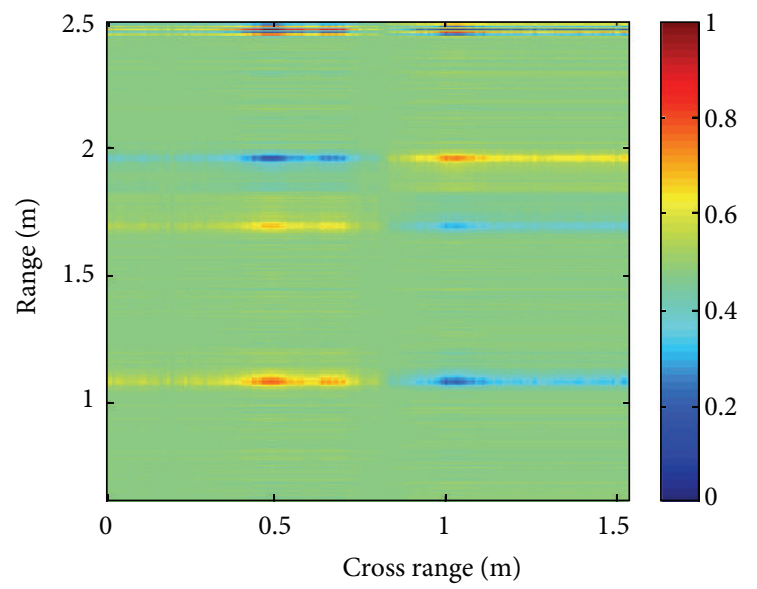

(b)

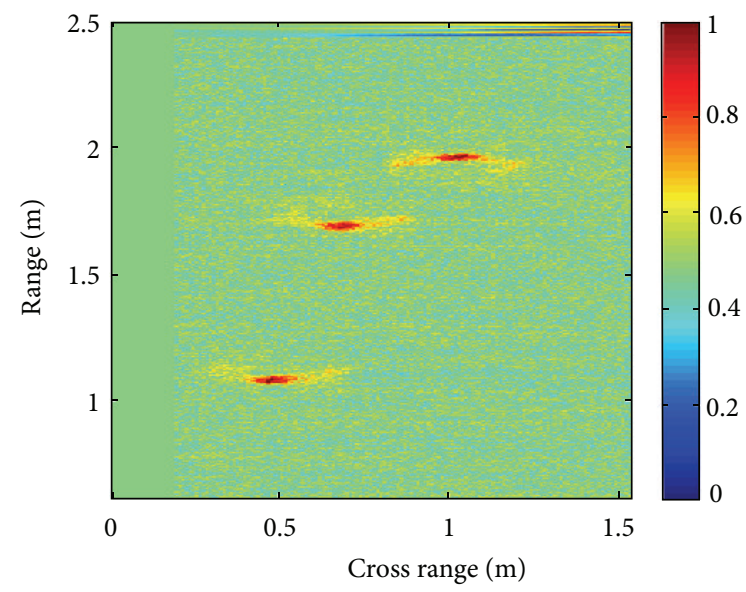

(d)

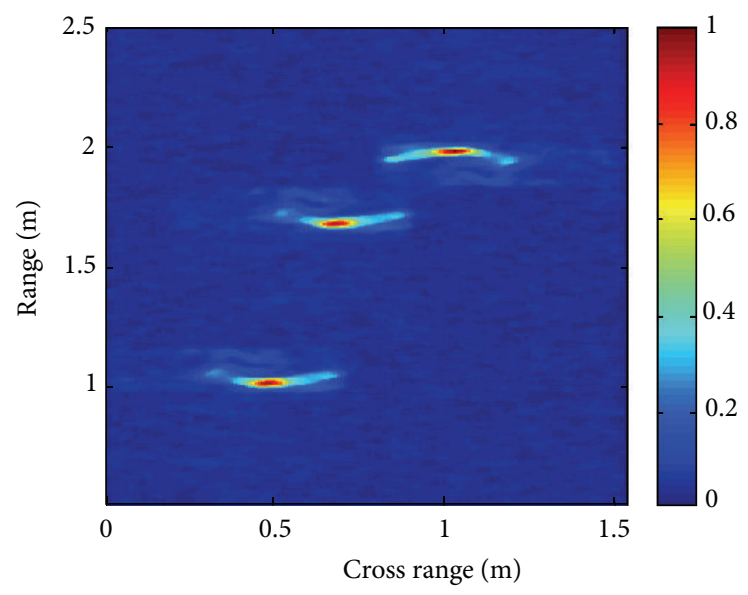

(e)

FIGURE 2: Image with three targets. (a) Original image. (b) SVD [11]. (c) Proposed fuzzy QRD (PIE). (d) Proposed fuzzy QRD (TS). (e) Background subtracted reference image. 


\section{Conflict of Interests}

The authors declare that there is no conflict of interests regarding the publication of this paper.

\section{References}

[1] M. G. Amin, Through the Wall Radar Imaging, CRC Press, Boca Raton, Fla, USA, 2011

[2] J. Moulton, S. Kassam, F. Ahmad, M. Amin, and K. Yemelyanov, "Target and change detection in synthetic aperture radar sensing of urban structures," in Proceedings of the IEEE Radar Conference (RADAR '08), pp. 1-6, May 2008.

[3] Y.S. Yoon and M. G. Amin, "Spatial filtering for wall-clutter mitigation in through-the-wall radar imaging," IEEE Transactions on Geoscience and Remote Sensing, vol. 47, no. 9, pp. 3192-3208, 2009.

[4] M. Dehmollaian and K. Sarabandi, "Refocusing through building walls using synthetic aperture radar," IEEE Transactions on Geoscience and Remote Sensing, vol. 46, no. 6, pp. 1589-1599, 2008.

[5] G. E. Smith and B. G. Mobasseri, "Robust through-the-wall radar image classification using a target-model alignment procedure," IEEE Transactions on Image Processing, vol. 21, no. 2, pp. 754-767, 2012.

[6] S. S. Ram, C. Christianson, Y. Kim, and H. Ling, "Simulation and analysis of human micro-dopplers in through-wall environments," IEEE Transactions on Geoscience and Remote Sensing, vol. 48, no. 4, pp. 2015-2023, 2010.

[7] C. Debes, Advances in detection and classification for through the wall radar imaging [Ph.D. Dissertation], Technische University Darmstadt, 2010.

[8] F. H. C. Tivive, M. G. Amin, and A. Bouzerdoum, "Wall clutter mitigation based on eigen-analysis in through-the-wall radar imaging," in Proceedings of the 17th International Conference on Digital Signal Processing (DSP '11), pp. 1-8, July 2011.

[9] M. M. Riaz and A. Ghafoor, "Principle component analysis and fuzzy logic based through wall image enhancement," Progress in Electromagnetic Research, vol. 127, pp. 461-478, 2012.

[10] M. M. Riaz and A. Ghafoor, "Through wall image enhancement based on singular value decomposition," International Journal of Antenna and Propagation, vol. 2012, Article ID 961829, 20 pages, 2012.

[11] P. K. Verma, A. N. Gaikwad, D. Singh, and M. J. Nigam, "Analysis of clutter reduction techniques for through wall imaging in UWB range," Progress in Electromagnetics Research B, vol. 17, pp. 29-48, 2009.

[12] A. N. Gaikwad, D. Singh, and M. J. Nigam, "Application of clutter reduction techniques for detection of metallic and low dielectric target behind the brick wall by stepped frequency continuous wave radar in ultra-wideband range," IET Radar, Sonar and Navigation, vol. 5, no. 4, pp. 416-425, 2011.

[13] M. M. Riaz and A. Ghafoor, "QR decomposition based image enhancement for through wall imaging," in Proceedings of the IEEE Radar Conference, pp. 978-983, 2012.

[14] G. H. Golub and C. F. Loan, Matrix Computation, John Hopkins, Baltimore, Md, USA, 1998.

[15] L. X. Wang, A Course in Fuzzy Systems and Control, Prentice Hall, Englewood Cliffs, NJ, USA, 1997.

[16] T. Kanungo, D. M. Mount, N. S. Netanyahu, C. D. Piatko, R. Silverman, and A. Y. Wu, "An efficient $k$-means clustering algorithms: analysis and implementation," IEEE Transactions on Pattern Analysis and Machine Intelligence, vol. 24, no. 7, pp. 881892, 2002.

[17] T. Takagi and M. Sugeno, "Fuzzy identification of systems and its applications to modeling and control," IEEE Transactions on Systems, Man and Cybernetics, vol. 15, no. 1, pp. 116-132, 1985.

[18] R. C. Gonzalez and R. E. Woods, Digital Image Processing, Prentice Hall, Englewood Cliffs, NJ, USA, 3rd edition, 2007. 

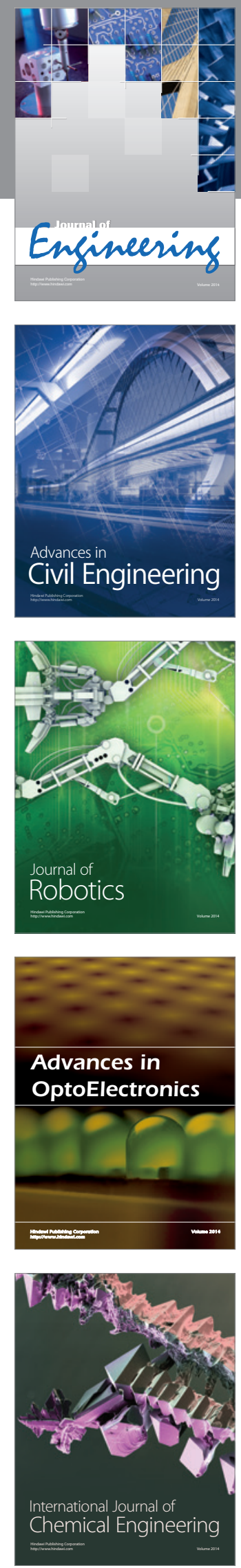

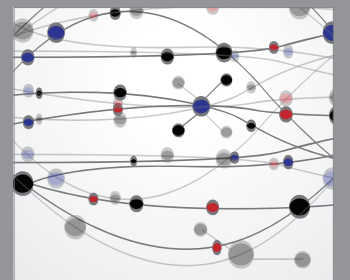

The Scientific World Journal
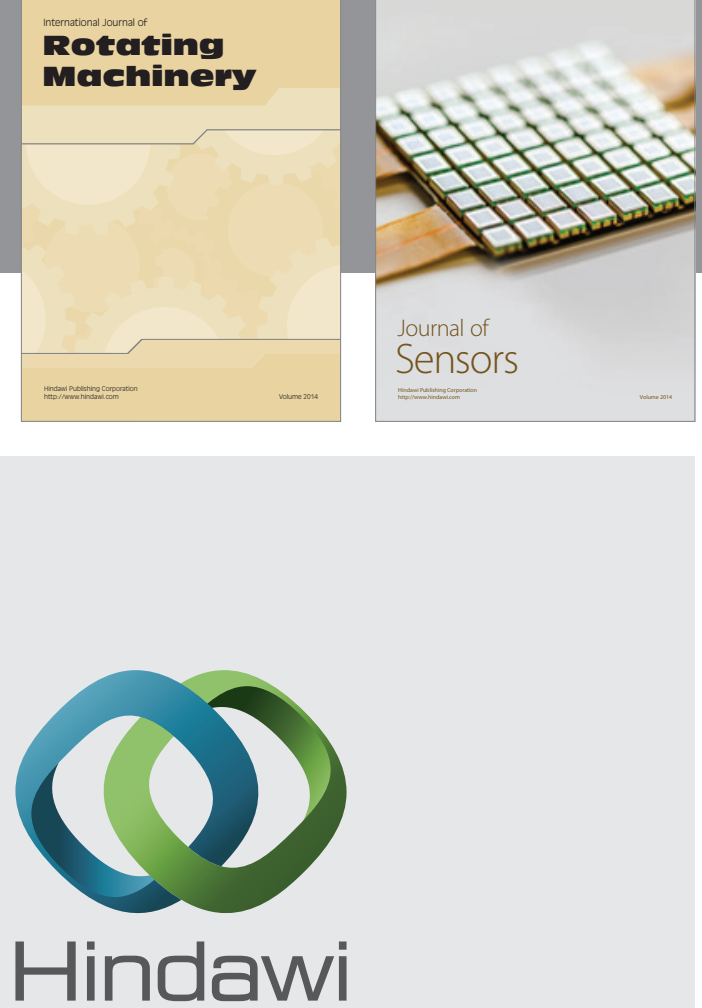

Submit your manuscripts at http://www.hindawi.com
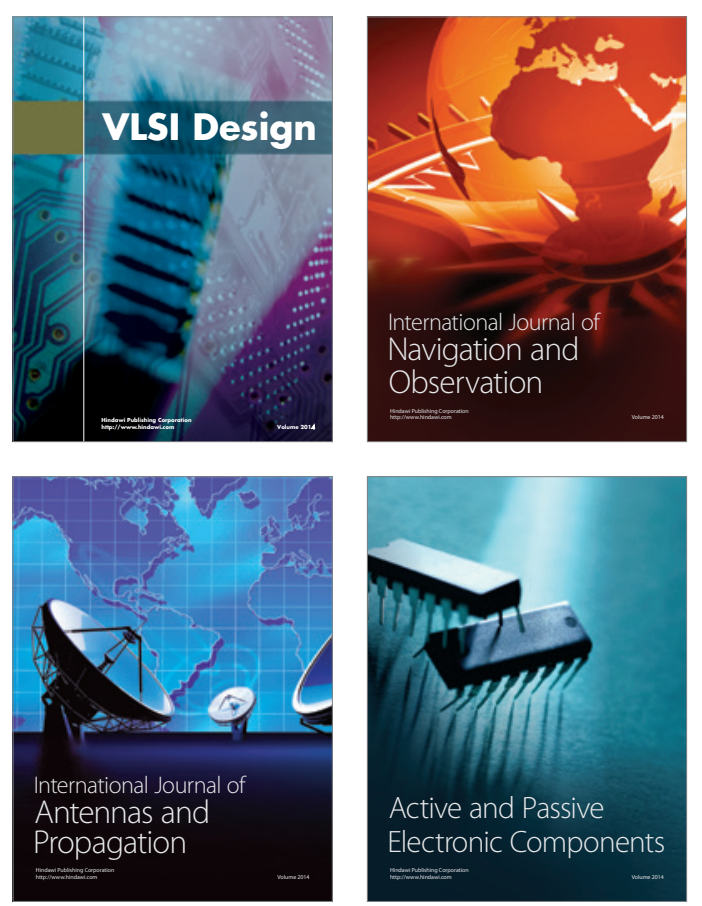
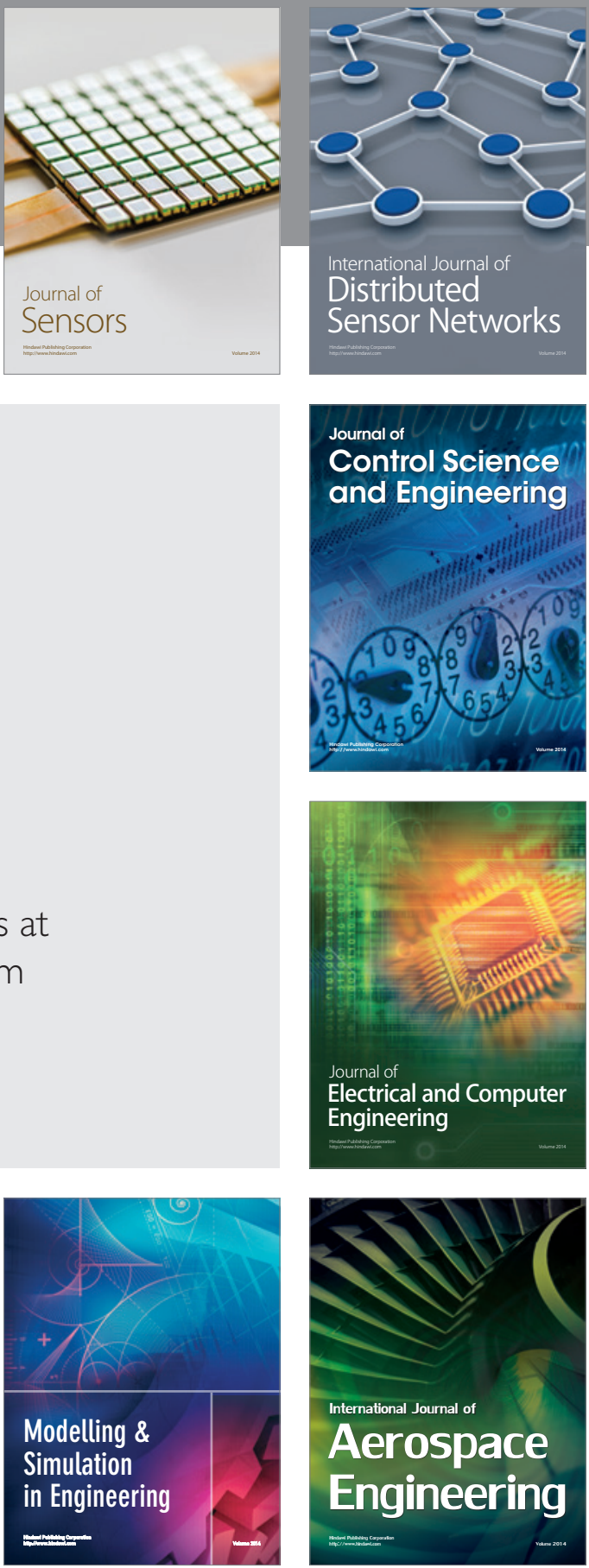

Journal of

Control Science

and Engineering
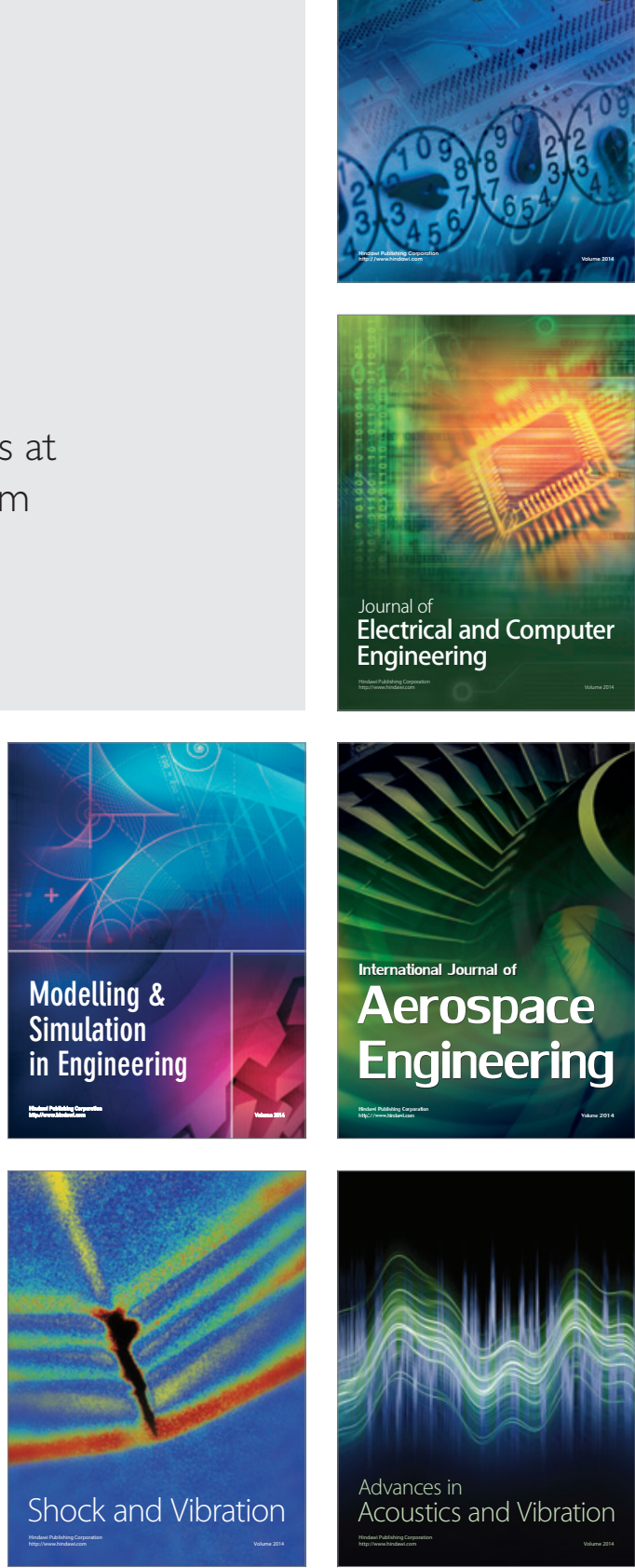